\title{
MeznSat-A 3U CubeSat for Monitoring Greenhouse Gases Using Short Wave Infra-Red Spectrometry: Mission Concept and Analysis
}

\author{
Abdul-Halim Jallad ${ }^{1, *}$, Prashanth Marpu ${ }^{2}$ D , Zulkifli Abdul Aziz ${ }^{1}$, Abdulla Al Marar ${ }^{3}$ and $^{-}$ \\ Mohammed Awad ${ }^{1}$ \\ 1 American University of Ras Al Khaimah, P.O. Box 10021, Ras Al Khaimah, UAE; \\ t.zulkifli@aurak.ac.ae (Z.A.A.); mohammed.awad@aurak.ac.ae (M.A.) \\ 2 Department of Electrical Engineering and Computer Science, Khalifa University, Masdar City, \\ P.O. Box 54224, Abu Dhabi, UAE; prashanth.marpu@ku.ac.ae \\ 3 UAE Space Agency, P.O. Box 7133 Abu Dhabi, UAE; A.AlMarar@space.gov.ae \\ * Correspondence: abdul.jallad@aurak.ac.ae; Tel.: +971-7-221500-1113
}

Received: 14 July 2019; Accepted: 27 September 2019; Published: 31 October 2019

\begin{abstract}
Climate change and global warming are attributed to the increased levels of greenhouse Gases in the atmosphere. Miniature low-cost, lightweight instruments on-board low-cost nanosatellite platforms such as CubeSats could be used to provide precise measurements of greenhouse gases levels. CubeSats, which usually have a narrow field of view, cost a fraction of what more expensive satellites with wide swaths cost. MeznSat is a $3 \mathrm{U}$ CubeSat that will carry a shortwave infrared (SWIR) micro-spectrometer as its primary payload, with the aim of deriving greenhouse gas concentrations in the atmosphere by making observations in the 1000-1650 nm wavelength region. The satellite, which is planned for launch in March 2020, is the result of a collaborative project between Khalifa University of Science and Technology (KUST) and the American University of Ras Al-Khaimah (AURAK) with a fund from the United Arab Emirates Space Agency (UAE-SA). The primary payload, Argus 2000, is a miniature, low-cost, space-qualified spectrometer that operates in the shortwave infrared (SWIR) bands. Argus 2000 is a ruggedized unit with a mass of less than $230 \mathrm{~g}$ and power consumption of less than $1 \mathrm{~W}$. Also, the Argus 2000 has 0.15 degrees viewing angle and $15 \mathrm{~mm}$ fore-optics. The secondary payload will consist of a high definition (HD) camera that will allow post-processing to achieve the high geolocation accuracy required for the SWIR spectrometer data. The RGB combination of visible and SWIR bands setup makes MeznSat a unique CubeSat mission that will generate an interesting dataset to explore atmospheric correction algorithms, which employ SWIR data to process visible channels. This paper describes the mission feasibility, mission analysis, design, and status of MeznSat.
\end{abstract}

Keywords: green-house gases; CubeSats; shortwave infrared (SWIR) spectrometer

\section{Introduction}

Increasing concentrations of Greenhouse gases in the atmosphere produced by human activities is the most significant driver of climate change [1]. The impacts of climate change are expected to negatively affect water quantity and quality in most arid and semi-arid areas [1]. Furthermore, low agricultural productivity throughout the tropics and subtropics is expected, accompanied by damage to ecosystems and biodiversity in these areas. The Abu Dhabi State of Environment Report 2017 highlighted key vulnerabilities associated with climate change, principally sea-level rise coastal flooding; increased salinity of coastal aquifers; impacts on the marine environment; heat stress; built environment impacts; more extreme weather events (floods, droughts, etc.); increased risk of dust storms; and risk from airborne contaminants (e.g., pesticides) [2]. 
Carbon dioxide and methane are the two most prevalent greenhouse gases. While methane does not last in the atmosphere as long as carbon dioxide, it is approximately 21-times more heat-absorptive than carbon dioxide per unit of weight [3]. This property makes methane 84-times more potent than carbon dioxide in the first twenty years following its release. Hence, both emissions (methane and carbon dioxide) have to be addressed and monitored in order to effectively reduce the impact of climate change.

Several algal bloom occurrences took place recently in the Arabian Gulf. Such Algal blooms can have a significant detrimental effect on the economy in the UAE. In addition, the Arabian Gulf hosts some of the largest desalination plants in the world, and most of them are located along the UAE coastline [1]. These desalination plants produce a significant percentage of the potable water consumed by UAE residents, and their desalination capacity is constantly increasing. Increased number of algal bloom occurrences in the UAE is threatening the optimal operation of these desalination plants, hence affecting water availability in the UAE. Effective monitoring and early sensing of algal blooms are required to manage the operations of the desalination plants efficiently. Algal blooms are also threatening the water quality in the coastal areas, thus becoming a health hazard. The algae produce toxins which (i) in small quantities make consumption of shellfish dangerous, and (ii) in large quantities decimate the fish population. Furthermore, other more common problems associated with harmful algal blooms include environmental damage, impact on tourism and commerce due to the repulsive green residue and its offensive odor [4]. An efficient and early sensing system is required to facilitate a swift response [1].

Algal bloom build-up is usually initiated from nutrient pollution, an overabundance of the essential plant nutrients nitrogen and phosphorus [4]. Sources of these elements are typically point sources (such as wastewater treatment plant discharges), non-point sources (such as septic tanks and stormwater runoff, urban areas and residential areas), and from nutrient-enriched rainfall or dust storms, which frequently occur in the region [4]. When the concentrations of nitrogen and phosphorus increase in a water body; the right combination of temperature, sunlight, and low flow can trigger an algal bloom [1,4]. While nitrogen and phosphorus occur naturally and are essential plant nutrients, an overabundance of these nutrients can cause significant imbalances in the water body's ecology, and blooms are one symptom [1,4].

The current research aims to explore and study the performance of the sensing in the shortwave infrared (SWIR) region (1000-1650 nm) in combination with the RGB camera to predict possible algal boom occurrences through estimation of the concentration of nutrients in the coastal waters of the Arabian Gulf [1]. Eventually, facilitating precautionary measures by the local authorities. Rather than using a single wavelength, the appropriate method for monitoring $\mathrm{N}$ content is by using spectral profiles to mitigate the effect of other mixtures. There are several indices defined for measuring nitrogen content in plants with a lot of success. However, not much work is identified to estimate nitrogen content in coastal waters. Nitrogen Indices are robust spectral transformations of two or more spectral bands, of which at least one is directly or indirectly related to Nitrogen content [5]. One commonly used index is the Normalized Difference Nitrogen Index (NDNI), which is a log10 transformed reflectance Nitrogen Index (NI) that is based Nitrogen's absorption feature at $1510 \mathrm{~nm}$ and a reference band at $1680 \mathrm{~nm}$ [5].

Furthermore, the SWIR region can also be used to map through aerosols, especially dust efficiently. Visible imagers are frequently incapable of seeing through fog, haze, smoke, and dust, contract to the capabilities of the longer wavelengths of the short-wave infrared that are more capable of penetration in such conditions and hence provide better imaging quality. At the same time, longer wavelengths in the SWIR region $(>2000 \mathrm{~nm}$ ) can be used to detect dust and characterize mineralogical information. Apart from this, the SWIR bands have been used for accurate atmospheric correction of visible and very near-infrared bands of sensors such as MODIS [6]. This experiment can also validate those measurements apart from providing complementary information to the information obtained from the 
camera on-board the proposed CubeSat and UAE satellites such as DubaiSat-1 [7], DubaiSat-2 [8], and KhalifaSat [9] images.

The primary payload of MeznSat is the Argus 2000 SWIR spectrometer, which is a miniature, low-cost, space-certified spectrometer that operates in the near-infrared bands (1000 nm to $1650 \mathrm{~nm})$ [1]. MeznSat's secondary payload is a High Definition camera that will allow post-processing to achieve the high geolocation accuracy required for the spectrometer. This setup of using visible and SWIR spectrometer together with a visible-optical imaging camera will generate an interesting dataset to explore atmospheric correction algorithms which employ SWIR data to process visible channels.

The MeznSat spacecraft bus design consists of an attitude determination and control system (ADCS), a power system, a Command and Data Handling (CDH) System, a communications system and the structure. The ADCS is required to achieve an accuracy of less than 1 degree due to the narrow Field of View (0.15 degrees) of the Argus 2000 spectrometer [10]. The ADCS uses three reaction wheels and three magnetic torquers for control, and sun sensors, a horizon sensor and a star tracker for attitude determination. The CDH consists mainly of an On-Board Computer board with an ARM9 processor, $64 \mathrm{MB}$ SDRAM and two SD cards for mass storage. The power system design includes 22 solar cells capable of producing 6.5 Watts of average power. The satellite's communications system consists of a UHF transmitter for the downlink, a VHF receiver for the uplink and a set of deployable dipole antennas.

The most relevant CubeSat projects include the CANX-2 mission [11] and the SathyambaSat mission [12]. CANX-2 is a $3 \mathrm{U}$ CubeSat mission that was launched in 2006 and uses the same spectrometer but does not carry an RGB camera on-board. SathyambaSat is a 2U CubeSat that was launched in 2016. SathyambaSat uses the ARGUS 1000 spectrometer as its only payload, and it has a moderate ADCS system, which relies on magnetic actuation [12]. While there are several similar missions reported in the literature, this is the first mission that uses Shortwave Infrared Spectrometer in combination with an RGB camera and a reactive attitude control system capable of less than 1-degree accuracy on a $3 \mathrm{U}$ form factor. The advantage of having the RGB Camera with the spectrometer is for geolocation.

In a previous paper, we provided an overview of the initial MeznSat design [1]. In this paper, we present a detailed description of the final MeznSat mission concept, analysis, and design, including a comprehensive overview of the spacecraft bus. The launch of MeznSat is expected to take place in March 2020 into sun-synchronous orbit with an altitude of $550 \mathrm{Km}$. Section 2 provides details on the mission concept, including orbital analysis, payloads used, and system-level design. Section 3 describes the different subsystems of the satellite, while Section 4 provides a summary of the mission analysis. The conclusions are provided in Section 5.

\section{Mission Concept}

This section provides an overview of the mission concept, including the mission aims, the payloads used, the platform selection, orbital analysis, and the mission's Concept of Operations (ConOps).

\subsection{Mission Objectives}

MeznSat mission objectives are as follows:

- Primary science: To monitor methane and carbon dioxide levels in the atmosphere using a SWIR spectrometer.

- Tentative science: To study the feasibility of combining SWIR sensing with RGB images to estimate the concentration of nutrients in the coastal waters to predict the possibility of algal blooms

- Educational: Provide educational experience to university students in the UAE in building and designing space missions. 


\subsection{Payloads}

The main scientific objective of this mission is to explore the performance of sensing in the shortwave infrared (SWIR) region and specifically in the range of $1000-1650 \mathrm{~nm}$, to detect the levels of methane and carbon dioxide in the atmosphere [1]. In order to fulfill this science objective, MeznSat carries two payloads; the primary payload is a SWIR spectrometer, and the secondary payload is an RGB camera. The combination of these two payloads provides a unique feature for MeznSat, allowing monitoring of GHG with the ability to perform geolocation using the RGB camera.

The chosen primary payload for this purpose is the Argus 2000 SWIR spectrometer (Figure 1). Argus 2000 is a miniature, low-cost, space-proven spectrometer that operates measuring only $4.5 \times 8 \times 8 \mathrm{~cm}$ in size. The instrument operates by dispersing light entering to be dispersed by a 300 groove $/ \mathrm{mm}$ grating and directed onto a 256-element Indium-Gallium-Arsenide detector array that is cooled by a Peltier cooler in order to reduce dark currents. The spectral range of the spectrometer is 1.0 to 1.65 micrometers, and its spectral resolution is 6 nanometers across 100 spectral channels. Gases within the spectral range of the Argus 2000 spectrometer include oxygen $(1.25 \mu \mathrm{m})$, carbon dioxide $(1.57 \mu \mathrm{m}$, $1.61 \mu \mathrm{m}$, and $2.05 \mu \mathrm{m})$, water $(900 \mu \mathrm{m}, 1.2 \mu \mathrm{m}$, and $1.4 \mu \mathrm{m})$, carbon monoxide $(1.63 \mu \mathrm{m})$, methane $(1.67 \mu \mathrm{m}$ and $2.25 \mu \mathrm{m})$, and hydrogen fluoride $(1.265 \mu \mathrm{m})$. Argus 2000 supports integration times between 0.5 and $4.096 \mathrm{~s}$ [13].

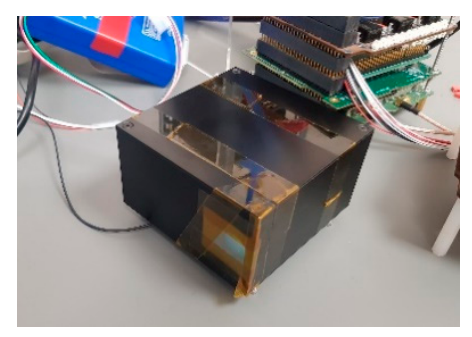

Figure 1. The Argus 2000 Spectrometer.

The Argus 2000 optical design consists of a telescope lens system, field stop and a primary parabolic mirror (with focal length and diameter of $35 \mathrm{~mm}$ ) to provide a collimated image of the surface tile into a surface grating with 300 groves per $\mathrm{mm}$. The spectrally divide image is reflected by the reflective grating onto another mirror that focuses the first spectral order of the surface tile onto the InGaAs detector [14,15].

The secondary payload is an RGB Camera that is based on a low voltage CMOS image sensor (Figure 2). The camera is developed by Tokyo University of Science (TUS) and has a significant flight heritage [1,16]. The camera utilizes the OV-9630 Image Sensor from Omnivision and uses a Microchip PIC microcontroller to interface between the image sensor and the on-board computer (OBC) [1]. A Universal-Asynchronous-Receiver-Transmitter is provided for communication with the OBC. The camera is capable of taking SXGA $(1280 \times 1024)$ and VGA $(640 \times 480)$ images. The camera has a small-footprint package of $45 \mathrm{~mm} \times 46 \mathrm{~mm} \times 15 \mathrm{~mm}$, weighs $39 \mathrm{~g}$ and has total power consumption of 0.11 Watts.

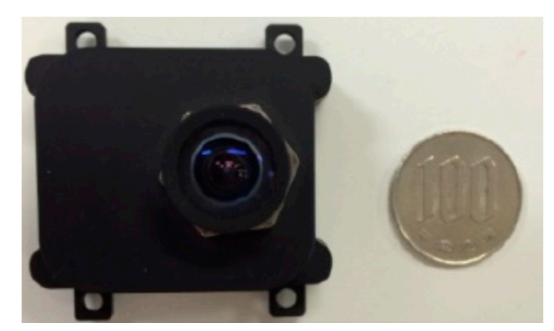

Figure 2. The RGB Camera used for MeznSat [16]. 


\subsection{Platform Selection}

After studying the possible options for the satellite platform, it was found that the minimum configuration that satisfies the proposed mission needs is the $3 \mathrm{U}$ CubeSat configuration. The $3 \mathrm{U}$ configuration has an equivalent size of three $1 \mathrm{U}$ CubeSats, as illustrated in Figure 3 . The standard size of a $3 \mathrm{U}$ CubeSat is $34 \mathrm{~cm} \times 10 \mathrm{~cm} \times 10 \mathrm{~cm}$. The main driving factor behind this decision is the volume that each platform provides to in-house all of the required subsystems. The small Field of View (FoV) of the Argus 2000 spectrometer dictates accurate pointing and hence a sophisticated ADCS, that would occupy at least $0.8 \mathrm{U}$. The payloads would require an estimated $1 \mathrm{U}$, which led to the decision of adopting a $3 \mathrm{U}$ platform for MeznSat.

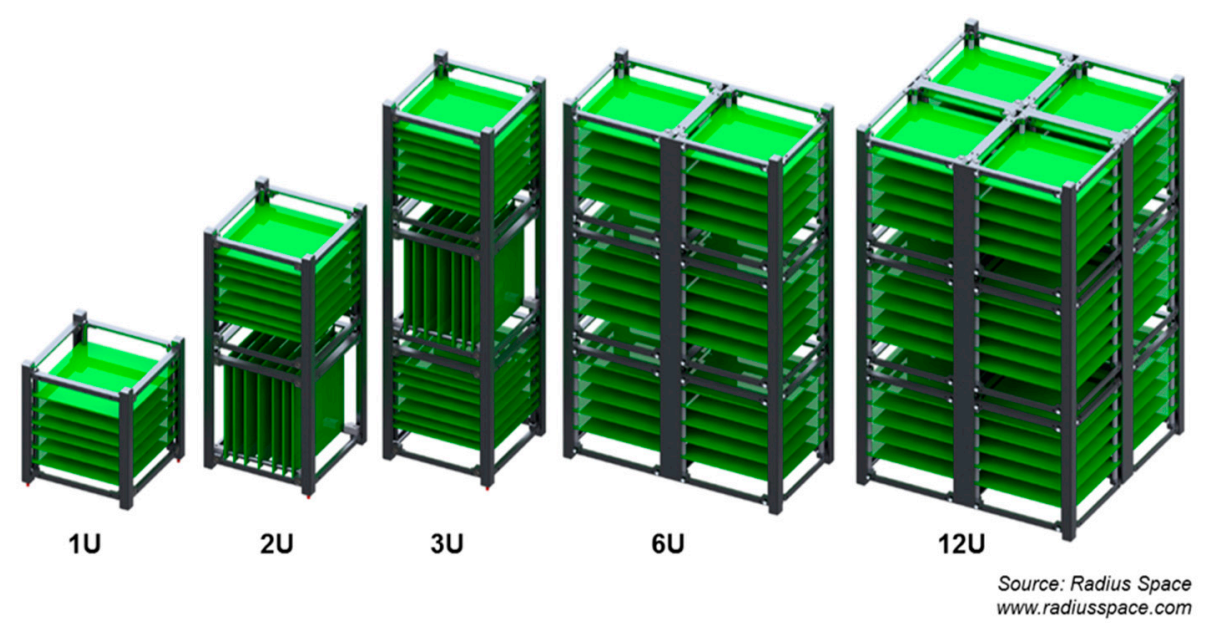

Figure 3. Illustration of the different CubeSat platforms.

\subsection{Concept of Operations}

MeznSat Concept of Operations (ConOps) is highlighted in Figure 4. During the satellite lifetime, it will operate in five main modes. In the launch and early checksum phase, the standard operations will be performed such antenna deployment, de-tumbling, and beacon transmission. Besides, all subsystems are checked for correct functionality during the initial checksum mode.

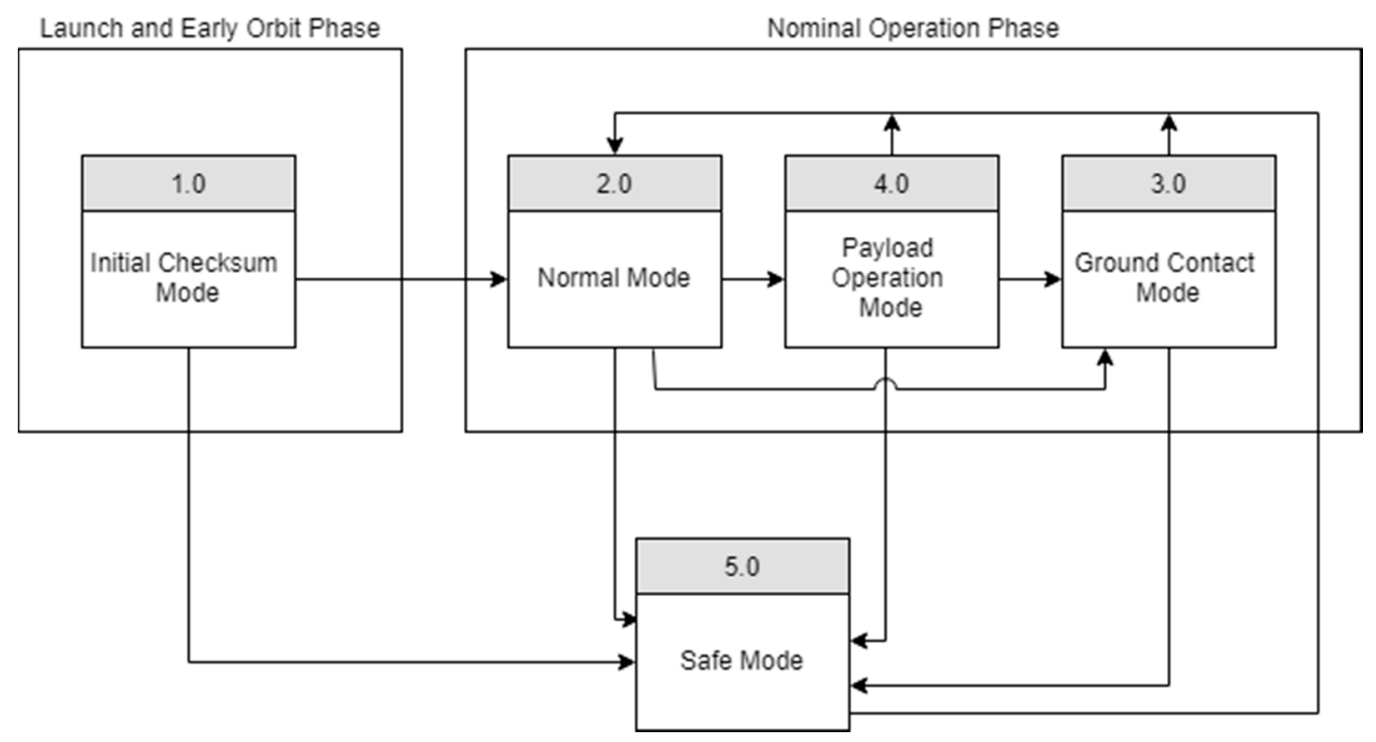

Figure 4. Overall modes of MeznSat operations. 
The nominal operation phase performs the main mission operations of the satellite, such as the payload operations. Normal mode is the mode in which the satellite will be in for most of its lifetime during which it will monitor all subsystem components and collect HK data while also sending a periodic beacon. In case of defects or abnormalities, the satellite enters the safe mode. Mission specific tasks like image capturing and storing image to memory are executed during payload mode. Ground Contact mode is entered when the satellite is in the range of the Ground Station during which it will be sending real-time data and receiving/executing commands.

\section{System Design and Platform Subsystems}

This section describes the system-level design of MeznSat and provides an overview of the subsystems used on-board. The spacecraft bus design includes five main subsystems that support the operation of the mission payloads. These are the Electrical Power Subsystem (EPS), the Attitude Determination and Control Subsystem (ADCS), the Command and Data Handling Subsystem (CDHS), the Telecommunications Subsystem (TS) and the mechanical subsystem. The overall system-level block diagram of MeznSat is shown in Figure 5.

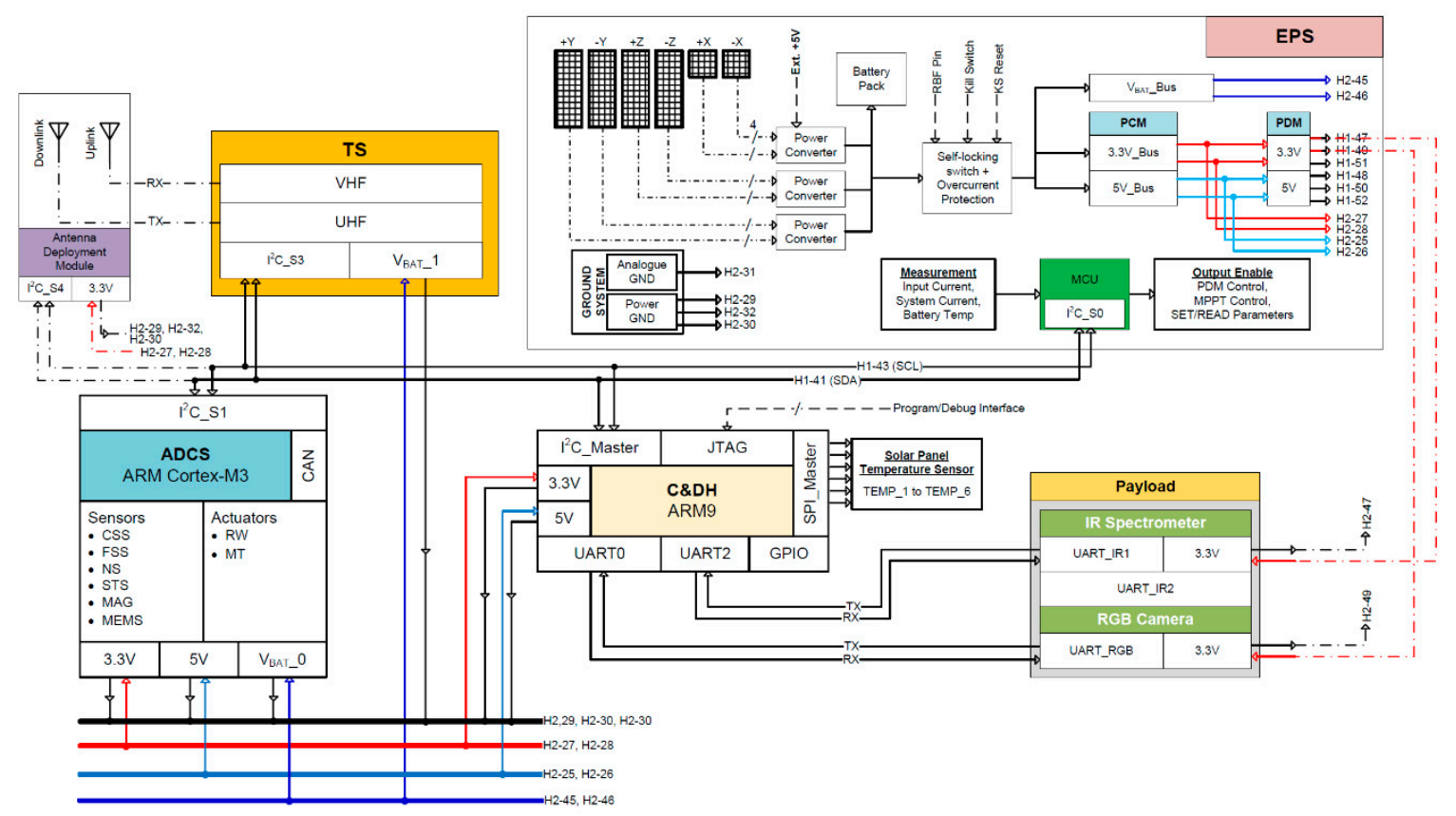

Figure 5. MeznSat system architecture.

\subsection{Mechanical Structure}

The mechanical structure is a $3 \mathrm{U}$ modular structure based upon the CubeSat standard from Innovative Solutions In Space (ISIS) [17]. It consists of three 1U PCB stacks that are contained within a secondary structure that forms the chassis of the structure that holds the PCBs in the stack (Figure 6). 


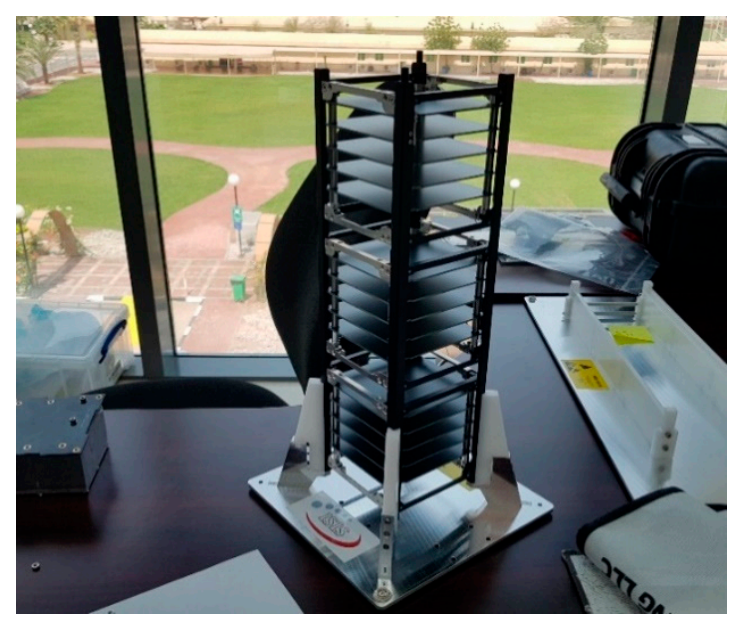

Figure 6. MeznSat mechanical structure.

\subsection{Telecommunications Subsystem (TS)}

The Telecommunications Subsystem consists of a UHF transmitter and VHF receiver. Four deployable dipole antennas are used for the UHF and VHF. The Transceiver provides the uplink and downlink communications between the satellite and ground stations.

A UHF transmitter is used for transmitting telemetry, science and image data from the satellite to the ground station. It transmits at $9.6 \mathrm{kbps}$ data rate using the BFSK modulation technique. The frequency range for the transmission carrier is from $430 \mathrm{MHz}$ to $450 \mathrm{MHz}$.

Table 1 shows the data volume generated by the payloads. The major portion of the payload data volume is generated from the RGB camera with 1,643,530 Bytes and 389,130 Bytes produced for SXGA format images and VGA format images respectively. Considering the SXGA format download a total of $22 \mathrm{~min}$ is required to download the payload data using the $9600 \mathrm{bps}$ UHF transmitter. STK simulations verify a total of $28 \mathrm{~min}$ per day Ground Station access time available for data download. It should be noted that these calculations do not consider using any on-board image compression.

Table 1. Data Volume Generated by Mission Payloads.

\begin{tabular}{ccc}
\hline Payload & \\
\hline RBG camera & SXGA & VGA \\
& $1,638,400$ bytes & 384,000 bytes \\
\hline Spectrometer (10 images) & 5130 bytes & 5130 bytes \\
\hline Total & $\mathbf{1 , 6 4 3 , 5 3 0}$ & $\mathbf{3 8 9 , 1 3 0}$ \\
\hline
\end{tabular}

A VHF receiver receives telecommands from ground station to the satellite. The receiver uses baseband modulator with $1.2 \mathrm{kbps}$ data rate and AFSK modulation. The frequency range for the transmission carrier is from $130 \mathrm{MHz}$ to $150 \mathrm{MHz}$.

\subsection{Attitude Determination and Control Subsystem (ADCS)}

The ADCS is the subsystem that is responsible for controlling the pointing of the satellite throughout the mission lifetime including de-tumbling the satellite after deployment, pointing the satellite as per the mission requirements and recovering it from any spin-ups. Given that the spectrometer used as the primary payload of the mission has a Field of View (FOV) of 0.15 degrees and to enable relatively accurate detection of GHG sources, a pointing accuracy of less than 1 degree is required. This requirement means that the passive attitude control mechanisms using magnetorquers usually used in CubeSats is not enough. Using reaction wheels and accurate attitude determination sensing (such as star sensors) would be required. 
The ADCS used for this mission is a CubeADCS 3-Axis integrated bundle from CubeSpace with a flight heritage of more than ten years (with the exception of the start tracker which has no flight heritage) [18]. Figure 7 shows an overview of the ADCS architecture and Figure 8 shows images of the ADCS hardware. The ADCS has three-axis control capability using three Reaction Wheels (with maximum momentum of $1.77 \mathrm{mNms}$ and a maximum torque of $0.23 \mathrm{mNm}$ ), in addition to three mangetorquers (to be used mainly for desaturation). The sensor set includes a deployable magnetometer, two CMOS cameras (one used for fine sun sensing and the other for horizon detection), ten coarse sun sensors and a star tracker based on ARM Cortex-M3 architecture.

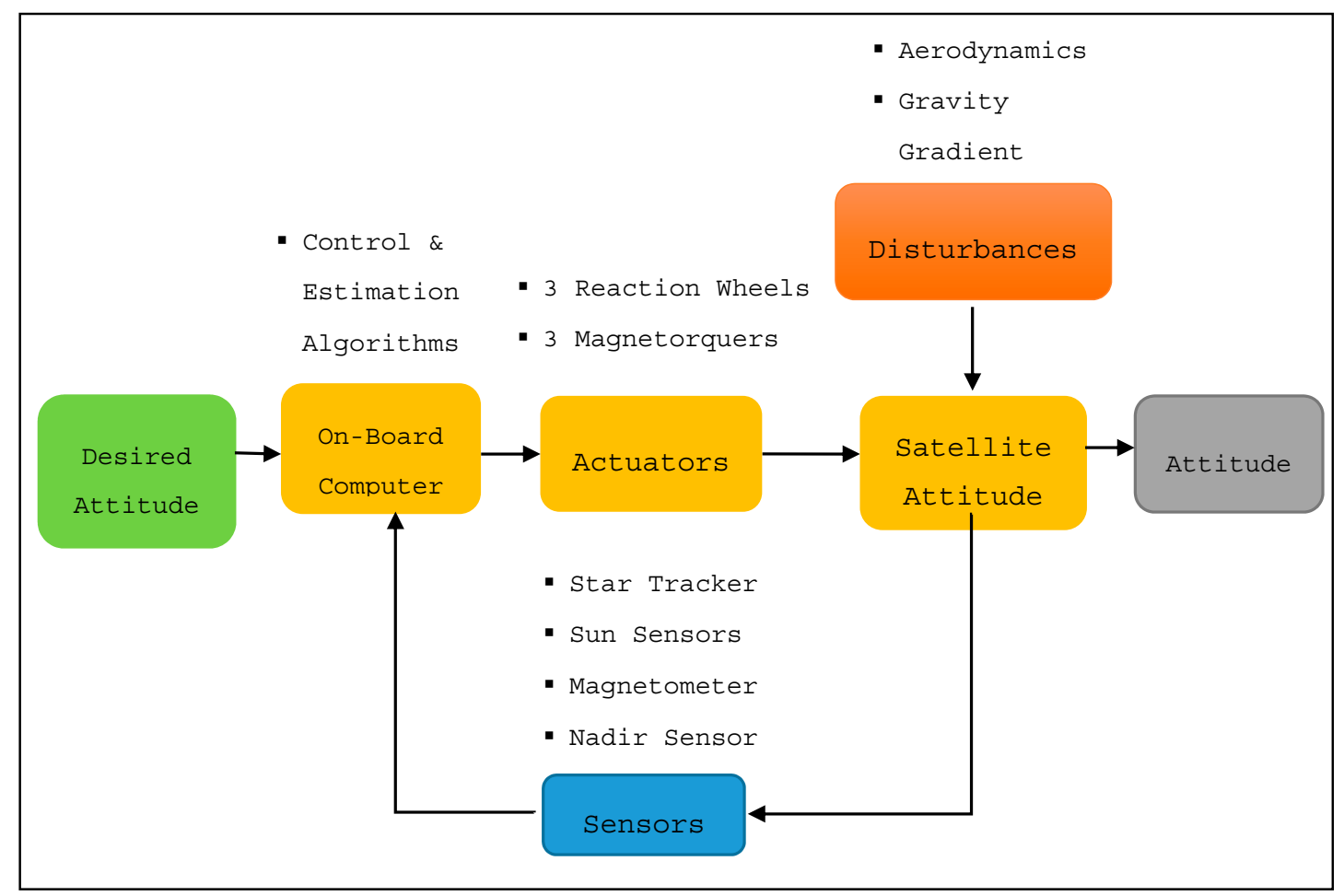

Figure 7. MeznSat Attitude Determination and Control Subsystem (ADCS) architecture.
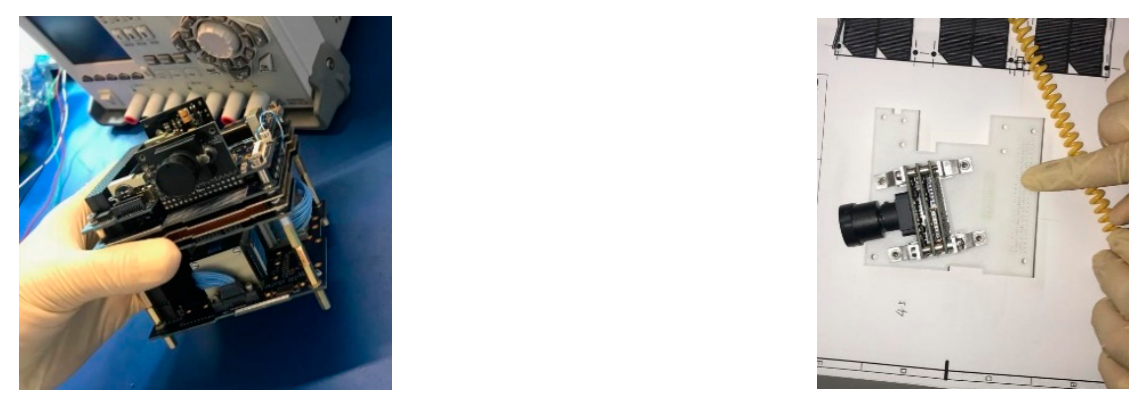

Figure 8. Integrated CubeADCS (left) and star tracker (right).

Figure 9 shows the complete set of ADCS modes for MeznSat. Due to the injection from the deployer, it is expected that MeznSat would experience rotation rate up to $10 \mathrm{deg} / \mathrm{s}$. "No Control Mode," where the ADCS does not perform active control, as per the CubeSat specifications document [11], which states that no operations need to be performed by the satellite for the first $30 \mathrm{~min}$ after deployment. Next, MeznSat will be controlled to move into "De-tumble/Attitude Acquisition Mode." The purpose of this mode is to reduce the rotation rate of MeznSat from $10 \mathrm{deg} / \mathrm{s}$ (worst case) to a slower angular rate of $<3 \mathrm{deg} / \mathrm{s}$. This will be followed by magnetometer deployment when ground contact with MeznSat has been established. As a result of the deployment, the Y-axis angular rate will increase. Y-Thomson 
Controller will reduce $\mathrm{X}$-axis and Z-axis angular rate and control $\mathrm{Y}$-axis angular rate to reach $<1 \mathrm{deg} / \mathrm{s}$. During payload operation, $X Y Z$-reaction wheel mode will be operational leading to decreasing the angular rate to $<0.03 \mathrm{deg} / \mathrm{s}$.

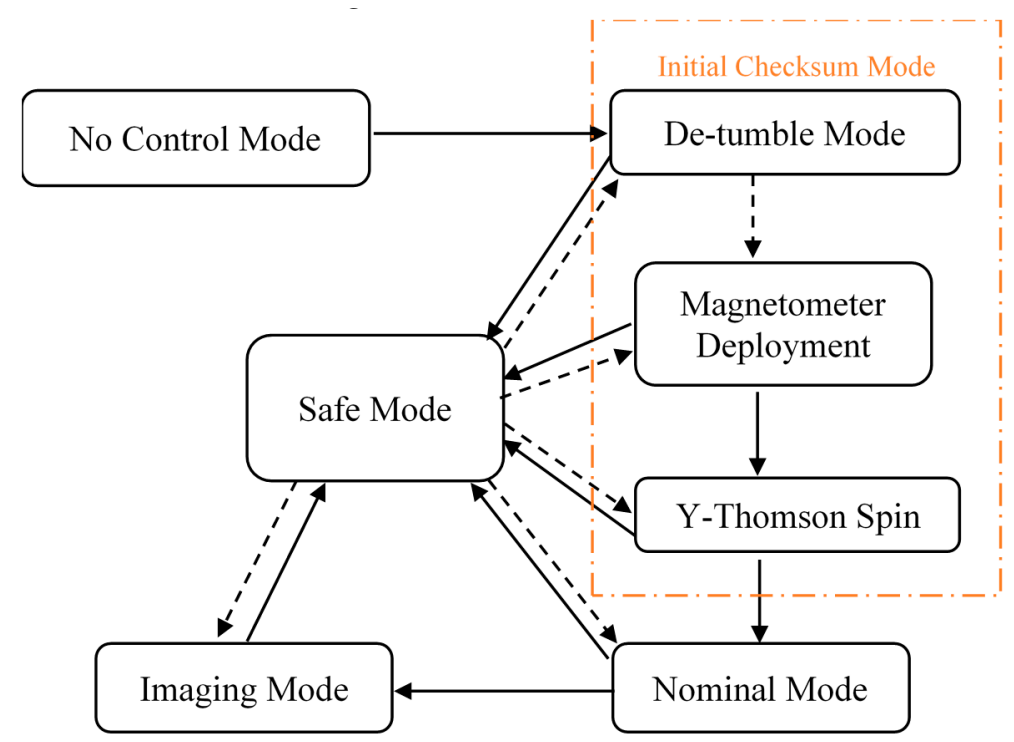

Figure 9. MeznSat ADCS modes of operation.

Changes between one mode to another are done autonomously (no ground intervention needed, except the magnetometer deployment and Y-Thomson spin). Exceptions are made for the satellite to recover from "Safe Mode" to any other mode depending on the satellite's angular rate only when the satellite receives commands from the ground station.

\subsection{Electrical Power Subsystem (EPS)}

The EPS provides electrical power to the spacecraft's different subsystems such that the satellite can function during the entire length of the mission. The power system will consist of three main components: the solar panels, the battery, and the power distribution system. Several options were considered for the solar panel configuration. The two most significant approaches suggested are the fixed solar panel configuration and the side deployable solar panel configuration. While the side deployable solar panel configuration appears to be appealing given the significantly higher power generation compared to the fixed solar panel configuration, power budget estimates (typical $6.24 \mathrm{Whr}$ and maximum $7.33 \mathrm{Whr}$ ) suggest that the fixed solar panel configuration will provide the required power necessary. A total of 22 solar cells are used to generate the satellite's power with the solar cell distribution around the structure being as shown in Figure 10. The power system is also equipped with a Li-Ion battery pack with a density of 38.4 Whr. The EPS can perform the Li-Ion battery charging without interrupting the payload operation. 


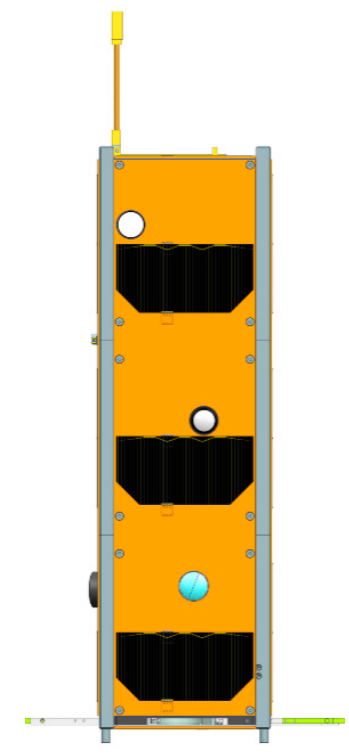

(a)

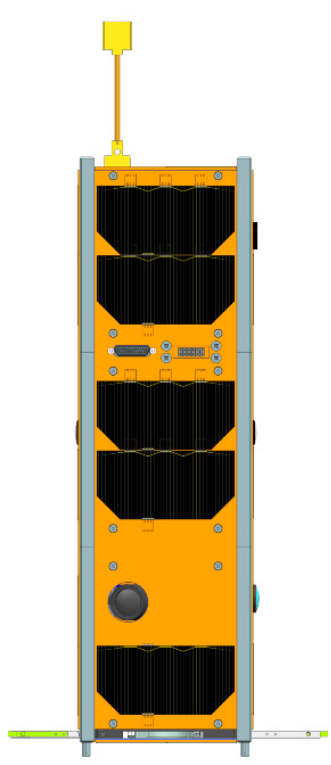

(b)

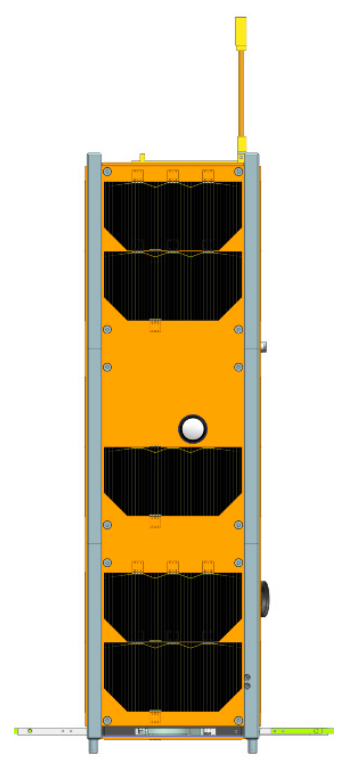

(c)

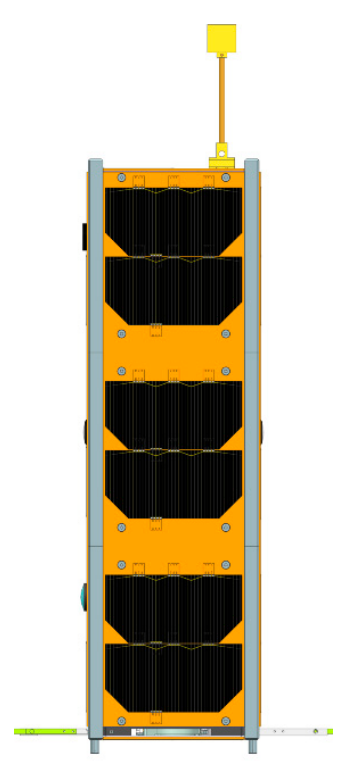

(d)

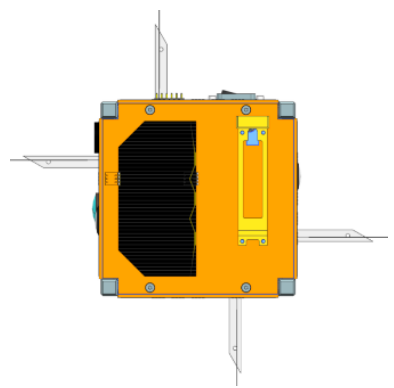

(e)

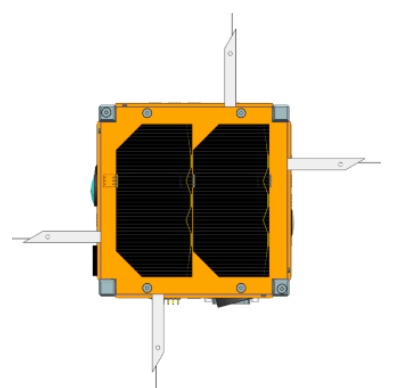

(f)

Figure 10. The solar cells distribution around the satellite structure: (a) +Z-axis; (b) +Y-axis; (c) -Z-axis; (d) -Y-axis; (e) + X-axis; (f) $-X$-axis.

\subsection{Command and Data Handling System (CDHS)}

The CDHS is responsible for running the flight software and with handling all data on-board the satellite. The central component of the CDHS is the on-board computer (OBC) which is based on a $400 \mathrm{MHz}$ ARM9 processor with $64 \mathrm{MB}$ RAM, 1 MB NOR flash for code storage, 256 KB FRAM for critical storage and two 2 GB microSD cards. I2C is used as the primary bus for the spacecraft bus, and UART is used for data handling between the OBC and the payloads.

\subsection{Flight Software}

The satellite flight operating software consists of several layers, as shown in Figure 11. On the very top of the hierarchy is the Mission Software, which executes MeznSats mission-specific. FreeRTOS [19] has been selected as the main operating system for the mission due to its lightweight, real-time performance and ease of use. The Hardware Abstraction Layer including (HAL) include all of the hardware-specific drivers and also includes a fail-safe FAT32 file system. 


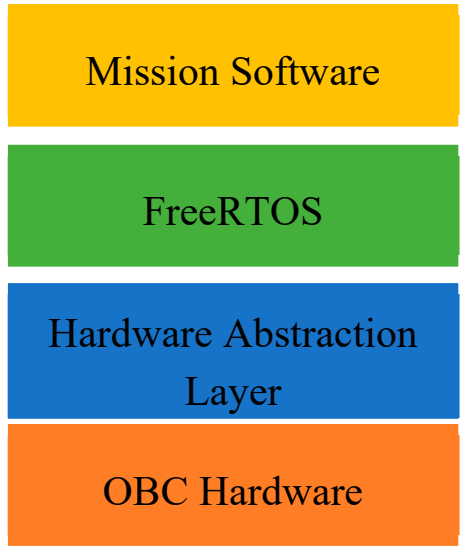

Figure 11. Flight software layers.

The software tasks in MeznSat have been categorized into four different classes:

(1) Tasks that run periodically: Include tasks that need to collect data from subsystems every number of minutes. These tasks include the Housekeeping collection task and the beacon transmission task

(2) Tasks that run based on a temporal value: Include tasks that need to run at a specific time. Temporal tasks include the ground contact task and the payload operation task.

(3) Tasks that run indefinitely: Tasks that are chosen to enter the running state because their delay is over, and they are the highest priority task within the ready pool of tasks. These tasks include the ADCS task and the normal mode task.

(4) Tasks that wait for events to happen: include tasks that are waiting on a semaphore or for data to be available on a queue. These tasks include the file management task, the command execution task, the safe mode task, and the initial checksum task.

The overall architecture of MeznSat software is shown in Figure 12.

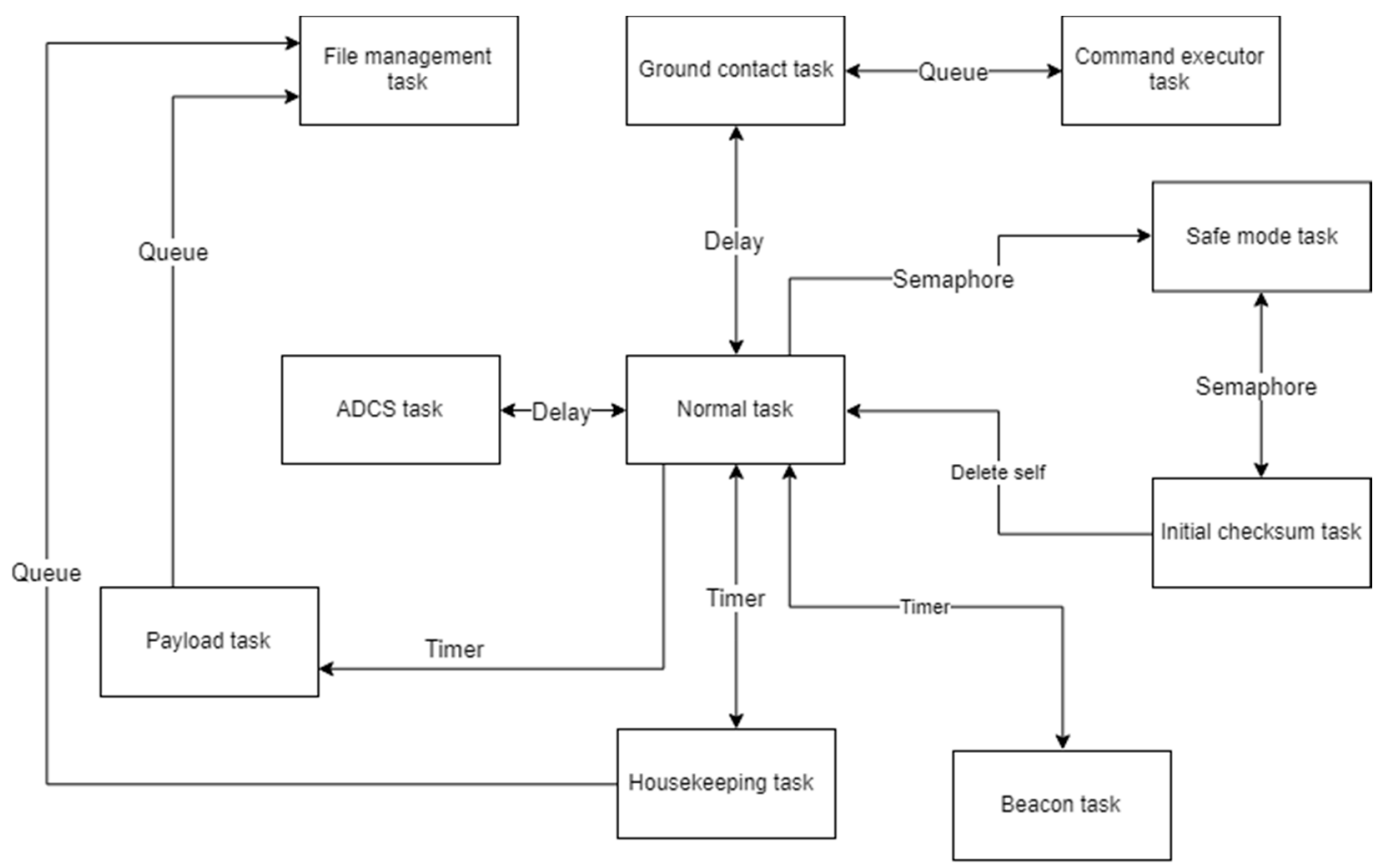

Figure 12. MeznSat software block diagram. 


\subsection{Atmospheric/Surface State Retrieval Algorithm}

Observations from the Argus 2000 spectrometer constitute a single sounding covering a ground sampling distance of approximately $1.5 \times 1.5 \mathrm{~km}$ from a $550 \mathrm{~km}$ altitude. Each sounding will be analyzed with a retrieval algorithm, which will fit the measured spectra by adjusting the unknown atmospheric, surface, and instrumental parameters. The retrieval consists of two parts: a forward model and a statistical comparison method [20]. The forward model is an approximate scheme to describe radiative transfer in the atmosphere, reflection by the surface, and the effects of the instrument on the incident radiation to generate detailed lookup tables [20]. Forward modeling will be performed using GENSPECT [21] software. GENSPECT is a radiative transfer modeling tool developed for CANX-2 mission.

The principal characteristics and flow of the retrieval algorithm are presented schematically in Figure 13. The initial step of the retrieval process begins by inputting an assumed environmental state that is defined by the surface pressure, surface reflectance, vertical temperature profile, mixing ratios of primary gases, water vapor, and other trace gases, in addition to cloud and aerosol optical depth distributions [20]. These parameters can be initialized from known climatology and additional satellite data products e.g., MODIS for surface reflectance, cloud, and aerosol optical distribution. This information is combined with pre-tabulated, wavelength-dependent gas, aerosol, and cloud optical properties. The gas absorption cross-sections for the spectral range are derived and tabulated using GENSPECT and spectral line databases such as HITRAN [22]. The synthetic spectra are then processed with a solar model and a model that simulates the instrument's spectral response to the incident radiation and produces results that can be compared directly to the calibrated spectra [20].

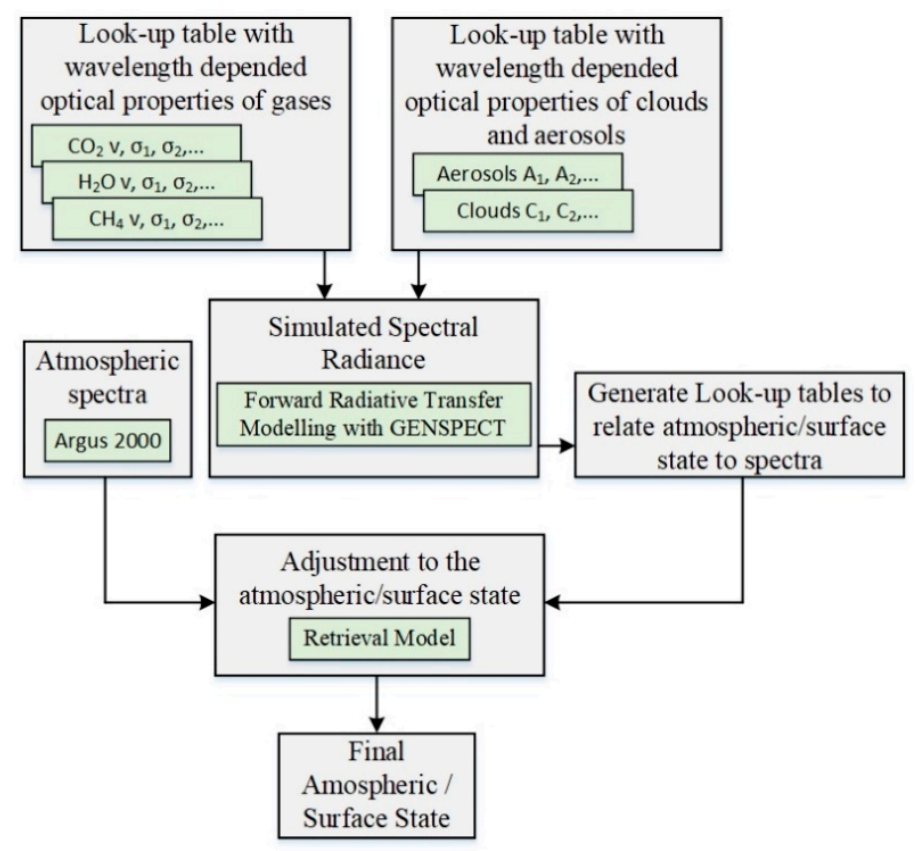

Figure 13. Atmospheric/surface state retrieval algorithm.

The comparison model compares the synthetic radiance spectra to the observations and uses an optimal estimation technique and produces a match to the measured spectra in the spectral range [20]. Finally, the atmospheric composition is derived from the best-match atmospheric state validated statistically.

To validate the results, data provided by the Total Carbon Column Observing Network (TCCON) [23] will be used. The satellite will acquire the data over the stations and the corresponding ground data acquired within \pm 30 min of satellite overpass time will be considered for comparison as in [24]. A portable SWIR spectrometer will also be used to accurately measure spectral characteristics of 
surface and downwelling radiation at a spectrally homogenous desert site in the UAE using systematic sampling to match the GSD. This data will be used to validate the instrument performance and for recalibration if needed.

\section{Results of Mission Analysis}

\subsection{Orbital Analysis}

The analysis for the orbit requirements for MeznSat took into consideration the following factors:

1. Orbit Lifetime-The minimum mission Lifetime required by the sponsor is three years.

2. Coverage time-Communication opportunity with the satellite using the mission's main ground station located at Khalifa University in Abu Dhabi.

3. Revisit-To calculate the MeznSat revisit over UAE in one day.

4. Payload operation opportunity-The opportunity for imaging over UAE.

All orbital analysis was done using Systems Took Kit (STK) [25]. Figure 14 shows that the altitude ranges between $45 \mathrm{~km}$ and $600 \mathrm{~km}$ satisfy the mission lifetime requirements (indicated by the orange ellipse on the figure), given the minimum mission requirements of three years and that the satellite is required to decay in less than 25 years as per the Inter-Agency Space Debris Coordination Committee (IADC) guidelines [26].

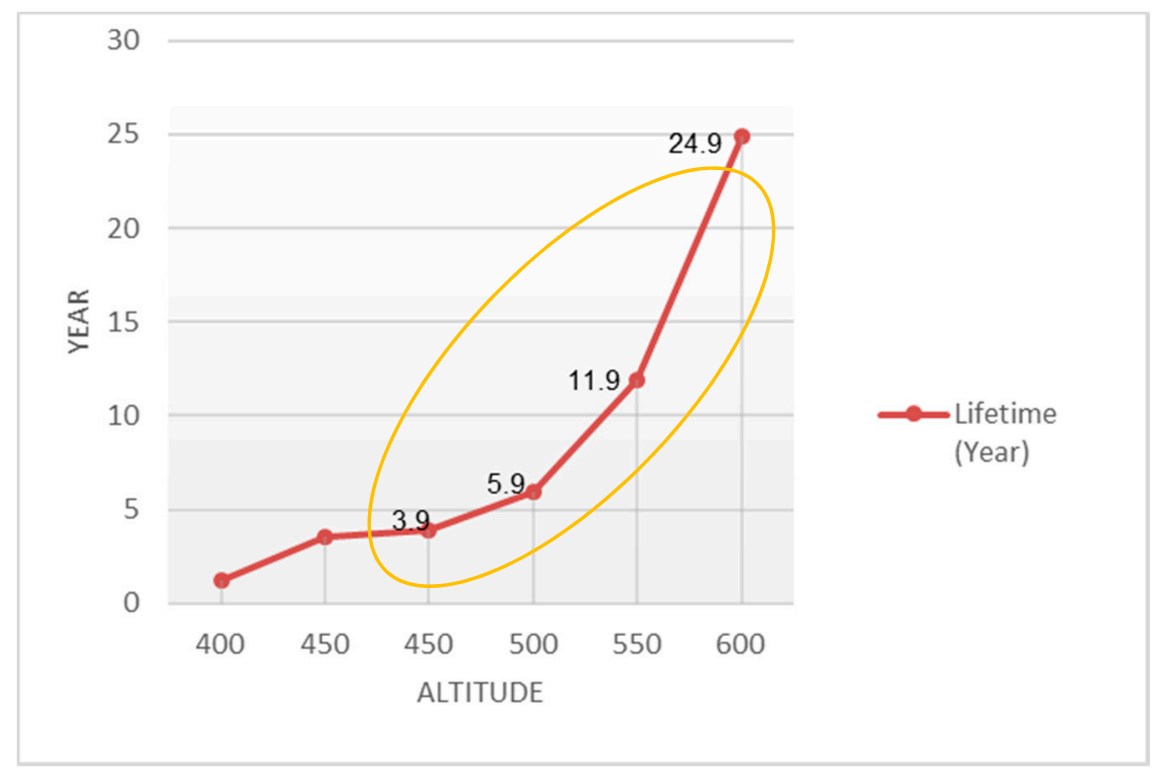

Figure 14. Lifetime analysis of MeznSat [1].

\subsection{Payload Coverage}

The payload coverage analysis was conducted to verify the coverage area above the UAE. The coverage analysis shown in Figure 15 was done based on the constraint that the sensors will only cover the area from 8 am to $5 \mathrm{pm}$. The grid area for the coverage in STK was set according to the ground sampling distance that the payload is capable of capturing i.e., $1.5 \mathrm{~km} \times 1.5 \mathrm{~km}$. The analysis showed that $46 \%$ of the total area of the UAE was covered by the spectrometer over the three-year mission period, given only nadir pointing satellite attitude. 


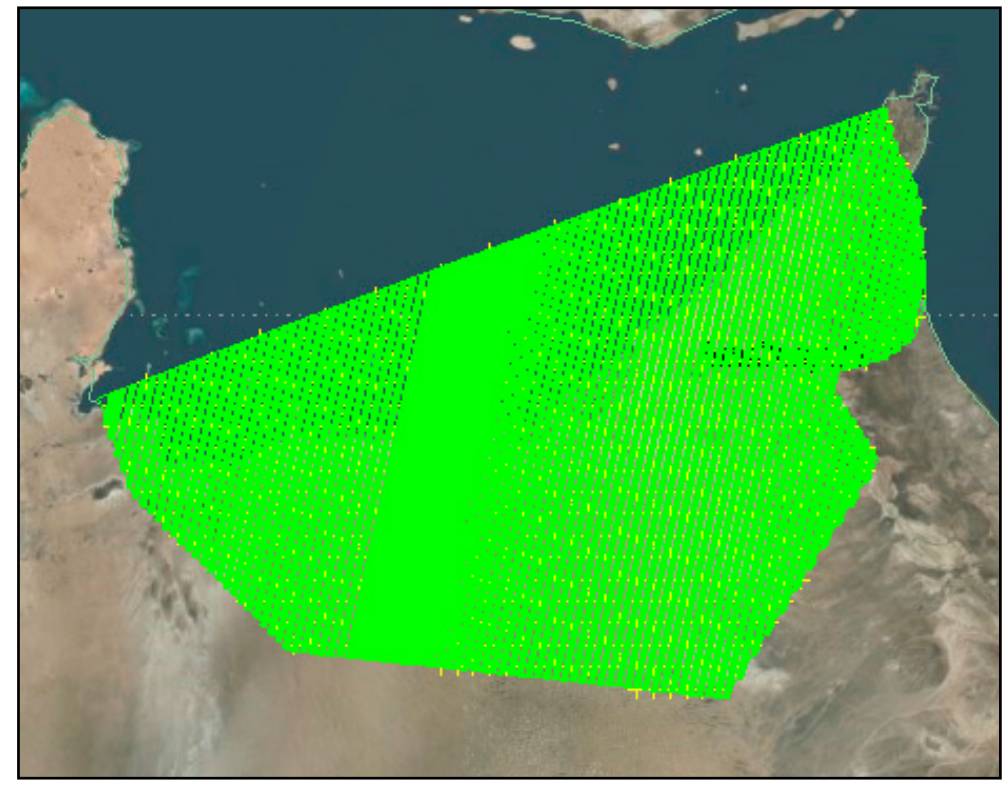

Figure 15. Spectrometer footprint coverage analysis for 36-month.

Average contact time for the satellite with the GS is approximately $10.40 \mathrm{~min}$ per passes for the SSO orbit with an altitude of $550 \mathrm{~km}$ and inclination 97 degrees.

\subsection{Volume, Mass, and Power Analysis}

A strong limiting factor that threatened the possibility of integrating the satellite into a $3 \mathrm{U}$ mission is the volume available for the components and subsystems. The internal system layout of and component arrangement of MeznSat is shown in Figure 16, with all subsystems fitting into the available $3 \mathrm{U}$ volume. A fit check was conducted using a 3D printed structure (while the main structure was under construction), and all components were found to fit as planned (Figure 17).

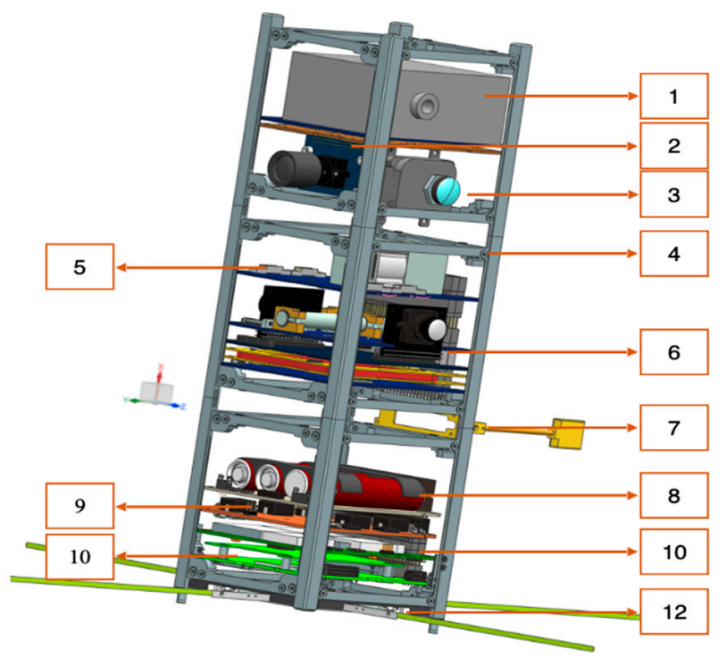

\begin{tabular}{|l|l|}
\hline No. & Component \\
\hline 1 & Spectrometer (Payload) \\
\hline 2 & ADCS Star Tracker \\
\hline 3 & RGB Camera (Payload) \\
\hline 4 & Main Structure \\
\hline 5 & 3 -axis Reaction Wheel \\
\hline 6 & 3 -axis ADCS Board \\
\hline 7 & Deployable ADCS Magnetometer \\
\hline 8 & Battery Board \\
\hline 9 & EPS Board \\
\hline 10 & Communication Board \\
\hline 11 & OBC Board \\
\hline 12 & Antenna \\
\hline
\end{tabular}

Figure 16. MeznSat internal system layout. 

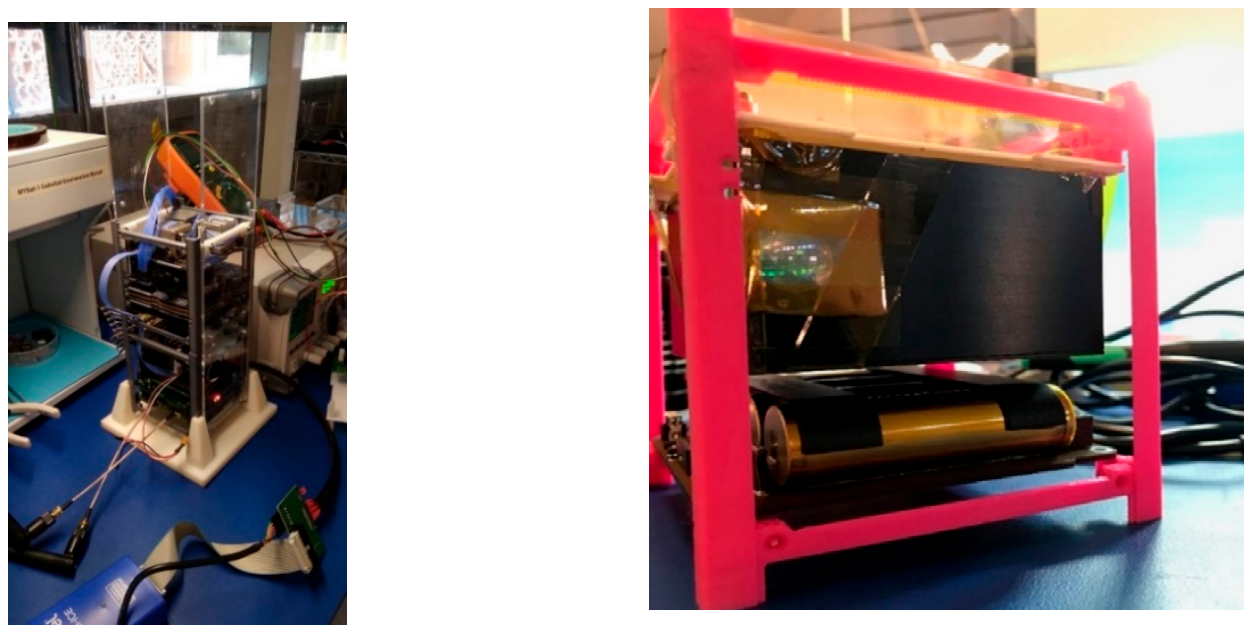

Figure 17. Fit check using 3D printed structures. Bottom $2 \mathrm{U}$ fitcheck (left) and top $1 \mathrm{U}$ fitcheck (right).

MeznSat mass budget is shown in Table 2. The results show that the satellite mass was found to be $2966 \mathrm{~g}$ with a mass margin of $26 \%$ from the maximum mass of $4 \mathrm{~kg}$ identified by the CubeSat Standard [27].

Table 2. MeznSat mass budget.

\begin{tabular}{|c|c|c|c|}
\hline No & Subsystem & Component & Mass (g) \\
\hline 1 & On-board computer (OBC) & Main + daughter board & 105 \\
\hline \multirow{2}{*}{2} & \multirow{2}{*}{ Communication } & Mainboard & 89 \\
\hline & & Antenna & 89 \\
\hline \multirow{4}{*}{3} & \multirow{4}{*}{ Electrical power subsystem (EPS) } & EPS Board & 105 \\
\hline & & CSS & 16 \\
\hline & & SP & 578 \\
\hline & & Battery Pack & 284 \\
\hline \multirow{4}{*}{4} & \multirow{4}{*}{ ADCS } & ADCS & 530 \\
\hline & & RW & 173 \\
\hline & & STS & 69 \\
\hline & & MAG & 16 \\
\hline \multirow{2}{*}{5} & \multirow{2}{*}{ Payload } & Spectrometer & 315 \\
\hline & & RGB Camera & 84 \\
\hline \multirow{2}{*}{6} & \multirow{2}{*}{ Structural } & Main Structure & 320 \\
\hline & & Screws, washers, and spacers & 55 \\
\hline \multirow{2}{*}{7} & \multirow{2}{*}{ Harness } & Cables & 105 \\
\hline & & Connectors & 33 \\
\hline \multicolumn{3}{|c|}{ Total mass with uncertainty } & 2966 \\
\hline \multicolumn{3}{|c|}{ Maximum mass allowable } & 4000 \\
\hline \multicolumn{3}{|c|}{ Mass Margin } & $26 \%$ \\
\hline
\end{tabular}

Figure 18 shows the simulated power profile of the satellite for one orbit. The figure shows a healthy power profile with a maximum expected battery Depth of Discharge of 5.5\%. The figure shows the generated power, the consumed power (based on the mission's power budget) and the battery profile. 


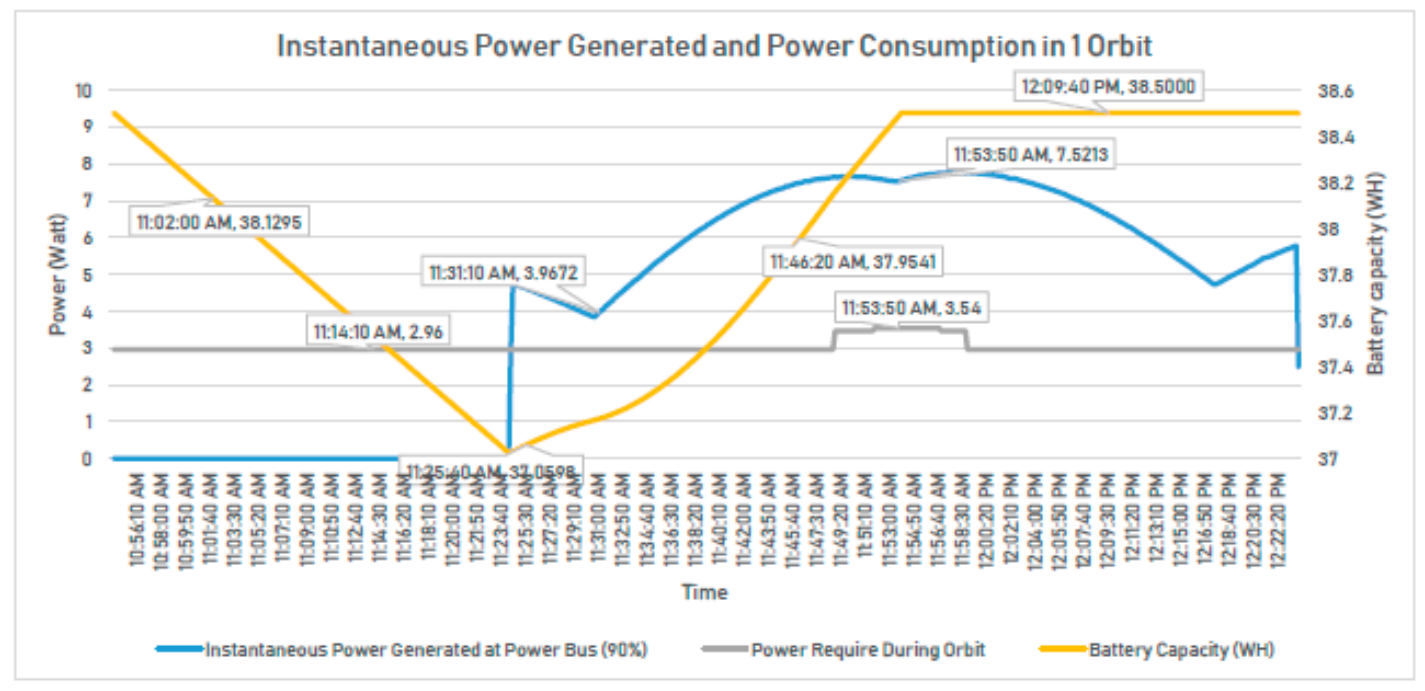

Figure 18. Power analysis for one orbit.

\subsection{Attitude Control Analysis}

After separation from the launch vehicle, the deployment mechanism will cause a tumbling rate applied to the spacecraft about $10 \mathrm{deg} / \mathrm{s}$. This rate needs to be de-tumbled to less than $3 \mathrm{deg} / \mathrm{s}$ in all axis, which will lead the spacecraft to the magnetometer deployment. To decrease the angular rate for all axis in the de-tumbling mode, Y-Magnetorquer actuator, and XYZ-axis rate sensors will be activated to achieve B-dot control mode and MEMS Rate estimation mode. Simulations were conducted using EOS Software [28] to verify the de-tumbling functionality of the ADCS. The results are shown in Figure 19. As mentioned earlier, Y-Thomson Controller will reduce $X$-axis and Z-axis angular rate and control Y-axis angular rate to reach $<1 \mathrm{deg} / \mathrm{s}$. Figure 20 shows the simulation results of the ADCS ins Y-Thomson mode. It will take the system $3 \mathrm{~min}$ to control the satellite to within $1 \mathrm{deg} / \mathrm{s}$ from an initial state of $3 \mathrm{deg} / \mathrm{s}$. A further detailed and comprehensive analysis and simulation of the ADCS was conducted, however, it is beyond the scope of this paper.

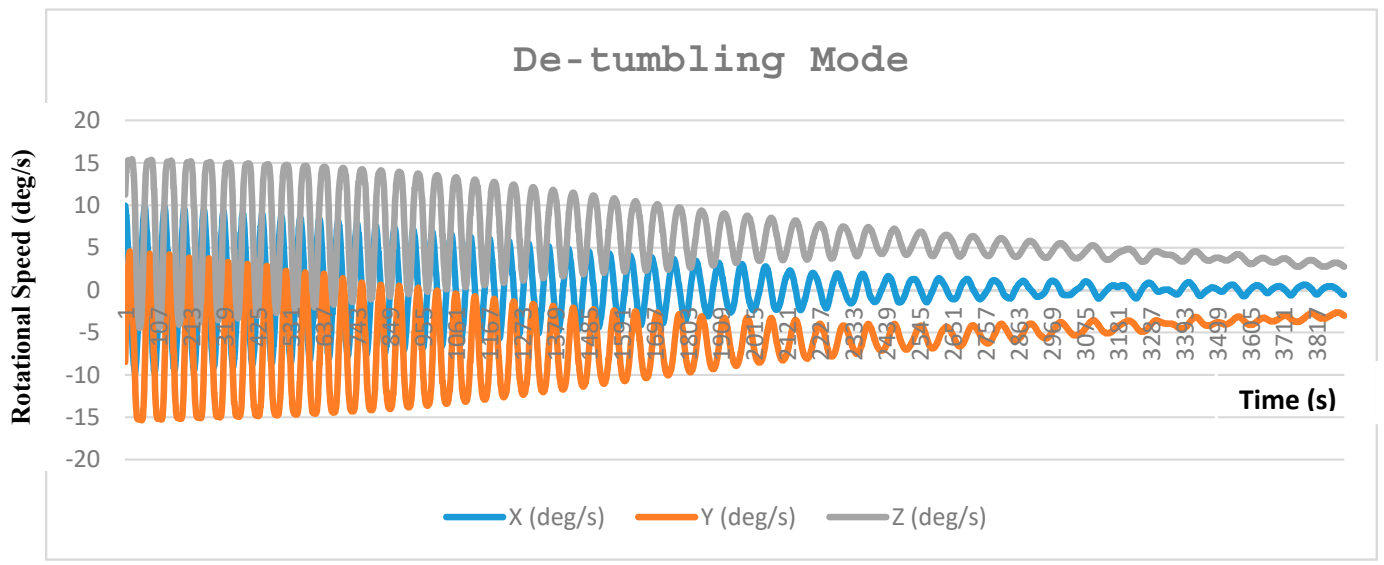

Figure 19. Results of the de-tumbling mode. 


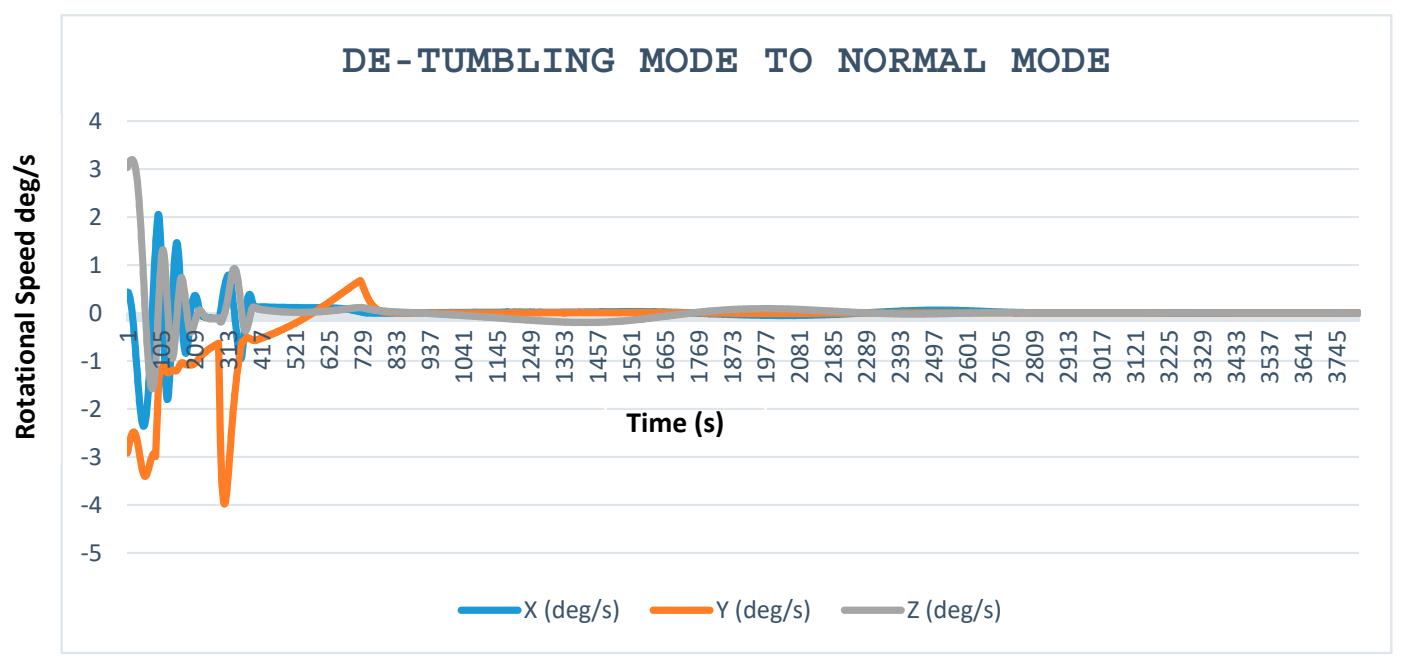

Figure 20. Results of the ADCS control using Y-Thomson controller.

\subsection{Thermal Analysis}

Thermal analysis using Finite Element Method (FEM) analysis was conducted using Siemens NX software [29]. The results produced indicate that the temperatures are within the operating temperature ranges for all of the components including the payload and the batteries. Extensive thermal testing will be conducted using a thermal vacuum chamber to verify the simulation results.

\section{Conclusions}

MeznSat is a 3U CubeSat that uniquely combines a SWIR spectrometer with an RGB camera for monitoring the level of GHG in the atmosphere. MeznSat payload is a SWIR spectrometer that will be operating in conjunction with an RGB camera to achieve high geolocation accuracy. Simulations show that the three-year mission will achieve $46 \%$ payload footprint coverage that can be leveraged using attitude maneuvering. The paper shows that the minimum possible platform to fulfill the missions' goals is a $3 \mathrm{U}$ platform given the current technology status for the various subsystems.

Available platform volume is the primary limitation found for the mission. It was a struggle to achieve an optimum design for the internal satellite configuration, given the dimensions of the various components, particularly the payloads and the ADCS. The mass budget shows a significant mass margin, and the power analysis shows a healthy power profile that is capable of handling additional pressure on the power system.

The small FOV of the spectrometer derives a requirement for an accurate ADCS system, requiring reaction wheels for actuation and sun sensors, star tracker and magnetometer for sensing, aiming for pointing accuracy of 1 degree. Simulations of the ADCS system shows appropriate performance that meets the stringent mission requirements.

Overall, the analysis presented in the paper included mass, volume, power, lifetime, payload coverage and pointing accuracy shows a feasible mission that uniquely combines SWIR payload with an RGB camera in a compact 3U platform. MeznSat is planned for launch in March 2020 into an orbit of $550 \mathrm{~km}$.

Author Contributions: Conceptualization, P.M., A.-H.J.; Writing-original draft preparation, A.-H.J., P.M., Z.A.A., M.A.; Writing—review and editing, A.-H.J., P.M., M.A., A.A.M.; Project administration, A.A.M., P.M., A.-H.J.; Software, A.-H.J., M.A.; Investigation, A.-H.J., P.M., Z.A.A., M.A.

Funding: This research was funded by the UAE SPACE AGENCY, grant number R01-2016-001.

Acknowledgments: The authors would like to thank all students involved as well as the UAE Space Agency for sponsoring the MeznSat program.

Conflicts of Interest: The authors declare no conflict of interest. 


\section{References}

1. Jallad, A.; Abdulaziz, Z.; Allam, A.; Marpu, P.; Tsoupos, A.; al Marar, A. MeznSat: A CubeSat for Greenhouse Gases Monitoring and Algal Blooms prediction. In Proceedings of the 32nd AIAA/USU Conference on Small Satellites, Logan, UT, USA, 4-9 August 2018. SSC18-WKIX-03.

2. Abu Dhabi State of Environment Report 2017. 2017. Abu Dhabi State of Environment. Available online: https://www.soe.ae/wp-content/uploads/2017/11/Climate_Change_English.pdf (accessed on 16 May 2019).

3. Greenhouse Gas Emissions. United States Environmental Protection Agency. Available online: https: //www.epa.gov/ghgemissions/overview-greenhouse-gases (accessed on 15 June 2019).

4. St. Johns River Water Management District. 2019. Available online: https://www.sjrwmd.com (accessed on 2 June 2019).

5. Herrmann, I.; Karnieli, A.; Bonfil, D.J.; Cohen, Y.; Alchanatis, V. SWIR-based spectral indices for assessing nitrogen content in potato fields. Int. J. Remote Sens. 2010, 19, 5127-5143. [CrossRef]

6. Wang, M.H.; Shi, W. The NIR-SWIR combined atmospheric correction approach for MODIS ocean color data processing. Opt. Express 2007, 15, 15722-15733. [CrossRef] [PubMed]

7. Al Rais, A.; Al Suwaidi, A.; Ghedira, H. DubaiSat-1: Mission overview, development status and future applications. In Proceedings of the 2009 IEEE International Conference: Geoscience and Remote Sensing Symposium (IGARSS 2009), Cape Town, South Africa, $12-17$ July 2009; Volume 5. [CrossRef]

8. Al Suwaidi, A. DubaiSat-2 Mission Overview. In Proceedings of the SPIE Remote Sensing, Edinburgh, UK, 24-27 September 2012. [CrossRef]

9. Japan Launches UAE's KhalifaSat and Climate-Monitoring Satellite into Orbit (20178 Space.com). Available online: https://www.space.com/42280-japan-khalifasat-gosat-2-satellite-launch-success.html (accessed on 20 May 2019).

10. Quine, B.M.; Jagpal, R.; Ho, A. Argus-A Canadian Pollution Monitor: Instrument Overview. In Proceedings of the 13th Canadian Astronautics Conference (ASTRO 2006), Montreal, QC, Canada, 25-27 April 2006.

11. Sarda, K.; Eagleson, S.; Caillibot, E.; Grant, C.; Kekez, D.; Pranajaya, F.; Zee, R.E. Canadian Advanced Nanospace eXperiment 2: Scientific and Technological Innovation on a Three-kilogram Satellite. Acta Astronaut. 2006, 59, 236-245. [CrossRef]

12. Rani, S.; Krishnamoorthy, N.; Harini, S.; Padmanabhan, K.; Singh, K. Sathyabama nanosatellite-Payload subsystem. In Proceedings of the Recent Advances in Space Technology Services and Climate Change 2010 Conference, Chennai, India, 13-15 November 2010.

13. Jagpal, R.K. Calibration and Validation of Argus 1000 Spectrometer-A Canadian Pollution Monitor. Ph.D. Thesis, York University, Toronto, ON, Canada, June 2011.

14. Jagpal, R.K. Calibration and in-orbit performance of the Argus 1000 spectrometer-The Canadian pollution monitor. J. Appl. Remote Sens. 2010, 4, 049501. [CrossRef]

15. Argus 2000 IR Spectrometer Owner's Manual. 2018. Available online: http://thothx.com/getmedia/4c0d3242b4fb-4e9d-abf7-85dbb5c6653f/20180815-Argus-2K-Owner-s-Manual,-Thoth-Technology,-rel-1-03.aspx (accessed on 30 August 2019).

16. Sugano, R.; Naohiro, U.; Shinichi, K.; Koji, W.; Kenji, N.; Kazuya, Y.; Tomohiro, N. Development of a Very Small High-Performance Image Acquisition System for Asteroid Exploration Rover MINERVA-II2. In Proceedings of the 65th International Astronautical Congress, Toronto, ON, Canada, 29 September-3 October 2014.

17. 3U CubeSat Structure. 2019. Available online: https://www.isispace.nl/product/3-unit-cubesat-structure/ (accessed on 16 June 2019).

18. 3-axis CubeADCS. Home Page. 2019. Available online: https://cubespace.co.za/3-axis/ (accessed on 31 August 2019).

19. FreeRTOS, Quality RTOS \& Embedded Software. 2019. Available online: http://www.freertos.org/ (accessed on 9 June 2019).

20. Natraj, V. Radiative Transfer Modeling for the Retrieval of $\mathrm{CO}_{2}$ from Space. Ph.D. Thesis, California Institute of Technology, Pasadena, CA, USA, 2008.

21. Quine, B.M.; Drummond, J.R. GENSPECT: A line-by-line code with selectable interpolation error tolerance. J. Quant. Spectrosc. Radiat. Transf. 2002, 74, 147-165. [CrossRef] 
22. Rothman, L.S.; Gordon, I.E.; Babikov, Y.; Barbe, A.; Benner, D.C.; Bernath, P.; Birk, M.; Bizzocchi, L.; Boudon, V.; Brown, L.; et al. The HITRAN 2012 Molecular Spectroscopic Database. J. Quant. Spectrosc. Radiat. Transf. 2013, 130, 4-50. [CrossRef]

23. TTCON. Home Page. 2019. Available online: https://tccon-wiki.caltech.edu/ (accessed on 30 August 2019).

24. Yoshida, Y.; Kikuchi, N.; Morino, I.; Uchino, O.; Oshchepkov, S.; Bril, A.; Saeki, T.; Schutgens, N.; Toon, C.G.; Wunch, D.; et al. Improvement of the Retrieval Algorithm for GOSAT SWIR XCO2 and XCH4 and Their Validation Using TCCON Data. Atmos. Meas. Tech. 2013, 6, 1533-1547. [CrossRef]

25. Analytical Graphics Inc. Home Page. 2019. Available online: https://agi.com/home (accessed on 14 July 2019).

26. UNOOSA; Inter-Agency Space Debris Coordination Committee. IADC Space Debris Mitigation Guidelines. Revision 1; IADC-02-01. September 2007. Available online: http://www.unoosa.org/documents/pdf/spacelaw/ sd/IADC-2002-01-IADC-Space_Debris-Guidelines-Revision1.pdf (accessed on 21 June 2019).

27. The CubeSat Program. 2014. CubeSat Design Specification Rev. 13. California Polytechnic State University. Available online: http://www.cubesat.org/s/cds_rev13_final2.pdf (accessed on 16 May 2019).

28. EOS Simulation Software Home Page. 2019. Available online: https://www.eosimulation.com/ (accessed on 10 June 2019).

29. Siemens NX. Home Page. 2019. Available online: https://www.plm.automation.siemens.com/global/en/ products/nx/ (accessed on 14 September 2019).

(C) 2019 by the authors. Licensee MDPI, Basel, Switzerland. This article is an open access article distributed under the terms and conditions of the Creative Commons Attribution (CC BY) license (http://creativecommons.org/licenses/by/4.0/). 